\title{
Weaning failure in a patient with bullous pemphigoid syndrome due to takotsubo syndrome
}

\begin{abstract}
Background: Takotsubo cardiomyopathy is a state mimicking MI which leads to impaired cardiovascular performance. Treatment is supportive without needs of inotropes. Although the onset is rapid it improves within a few days and normalizes in 2 months. That is why in hospital mortality and complications are low.

Case presentation: We report a case of Takotsubo cardiomyopathy in a woman who admitted the hospital with dyspnea and clinical signs of LHF. The patient was under treatment with prednisolone for bullous pemphigoid, a systemic autoimmune disease that rarely (10-15\%) combined with paraneoplastic tumors. After intubation the patient was unable to wean from mechanical ventilation. All clinical and investigation findings describe a type of non-ischemic cardiomyopathy with sudden but temporary weakening of the muscular portion of the heart, AHF, lethal ventricular arrhythmias and ventricular rupture also known as broken heart syndrome.

Conclusion: Updated AHA CPR guidelines 2015 recommendations for Post-Cardiac Arrest Care first include emergency coronary angiography for all hemodynamically or electrically unstable patients with ST elevation. Takotsubo cardiomyopathy can mimic MI on the onset and lead to respiratory failure because of impaired cardiovascular performances but treatment is supportive. That is why it can be misdiagnosed.
\end{abstract}

Keywords: Impaired cardiovascular performance, Weaning failure from mechanical ventilation, Non-ischemic cardiomyopathy
Volume 5 Issue 6 - 2016

\author{
Charalampos Martinos, Natasa Zagorianou, \\ Michael Tousert, Valdemar Viltsinski, \\ Vasileios Bekos, Georgia Karabela, Georgios \\ Katsimagklis, Panagiotis Strempelas, \\ Christoforos Komporozos, Emmanouel \\ Kasotakis \\ Department of Critical Care Medicine, Naval Hospital of \\ Athens, Greece
}

\begin{abstract}
Correspondence: Charalampos Martinos, Assistant Director of Intensive Care Unit of Naval Hospital of Athens and Sientific advisor of Cardiac Surgery Intensive Care Unit of Army Forces, 40I General Military Hospital of Athens, 16 Samou str. 15344 Gerakas, Athens Greece, Tel 00302106049725, Fax 00306937272595, Email 3damartin@gmail.com
\end{abstract}

Received: August 22, 2016 | Published: October 24, 2016

\section{Introduction}

Impaired Cardiovascular performance is one of the main factors associated with prolonged weaning from mechanical ventilation. ${ }^{15}$

The other factors are:

a. Systemic (chronic diseases, comorbidities, nutrition, metabolic disturbances and severity of illness),

b. Neurologic (critical illness neuromuscular abnormalities),

c. Respiratory (unresolved causes of respiratory failure, imbalance between work of breathing and respiratory muscle capacity and upper airway obstruction),

d. Treatment complications (ventilator-associated pneumonitis and infections, barotrauma, tracheostomy and sedation),

e. Treatment settings (weaning protocols, staffing and staff training) and finally

f. Cognitive (delirium, depression, anxiety and sleep deprivation).

\section{Case report}

A 70 year old woman admitted to the hospital complaining for dyspnea and low foot edema for the last 15 days. From her history she was diagnosed since Nov 2011 for bullous pemphigoid syndrome and was under medication with prednisolone. When she arrived she was hypoxemic (P1) that corrected with the use of oxygen at 2 lt. renal cannula. The rest of the tests were normal except the lung CT-scan that was a picture of interstitial pulmonary fibrosis (P2). There was a similar picture during $9 / 15$ that was talking about signs of COPD and pneumonic hypertension as well.

\section{A heart ECHO was normal (EF: 60\% ).}

The other morning she found under arrest but recovered immediately after the first cycle of CPR. She transferred intubated under sedation and mechanical ventilation to the Cardiac $\mathrm{CU}$. The ABGs were pH: 7,15/PO: 89,2/PCO : 72,7/ $\mathrm{HCO}_{3}: 25,8 / \mathrm{SaO}_{2}$ : $89,5 \% / \mathrm{FiO}_{2}: 1$. There was a new ST-segment at the ECG (P3) and troponin-I levels less than 0,1 the first day and 9,17 the other morning, both correlated with the arrest episode. A new heart ECHO found LV dilatation and PASP about $70 \mathrm{mmHg}$. After a medical council they decided that it should be better for her to move to the ICU and treated as lung infection increasing the prednisolone doses. There was a rapid decrease of ventilator support (CPAP/PS: 10) and FiO2 (40\%) that made us discontinue safe sedation to check her neurologically. For our surprise she was rapidly fully awake but unable to wean from mechanical ventilation, following our protocol, because she was hemodynamicaly unstable with the need of high doses of norepinephrine. The angiography showed absence of obstructive coronary disease and following Mayo criteria the diagnosis of Takotsubo syndrome was set. We decided then to continue ventilation support in order to minimize the work of breath (tracheostomy) and the use of hemodynamic support (vasoconstriction) as well as checking the heart function by ECHO and the heart rhythm because of ventricular arrhythmias. Markers for tumors or neuromuscular diseases were also send without any findings. A bronchoscopy (P4) for washing-brushing was also done looking for a paraneoplastic tumor. Biopsies of the lung were taken for more investigation in order to prove theory. Nothing of the above proved unless a subclinical LID. 6 weeks later the patient remains connected to the ventilator (PS: 6/ PEEP: 4), without vasopressors support and the heart systolic function normal again. 


\section{Discussion}

The typical presentation of Takotsubo cardiomyopathy is a sudden onset of congestive heart failure associated with ECG changes mimicking a myocardial infarction of the anterior wall. The term is taken from the Japanese name for an octopus trap (tako tsubo), which has a shape that is similar to the systolic apical ballooning appearance of the LV in the most common (apical ballooning cardiomyopathy/ syndrome). ${ }^{4,5}$ The first studied case of Takotsubo cardiomyopathy was in Japan in 1991 by Sato et al. The syndrome finally occurred in 1997 when Pavin et al. wrote about two cases of "reversible LV dysfunction precipitated by acute emotional stress."

The cause of Takotsubo cardiomyopathy is not fully understood, but several mechanisms have been proposed as:
a. Wraparound $\mathrm{LAD}^{6,7}$
b. Transient vasospasm. ${ }^{8-10}$
c. Microvascular dysfunction and
d. Mid-ventricular obstruction, apical stunning. ${ }^{11}$

Stress is the main factor in Takotsubo cardiomyopathy, with over $85 \%$ of cases are set in motion by either a physically or emotionally stressful event that prefaces the start of symptoms (stress-induced cardiomyopathy). ${ }^{2}$

\section{Drugs $^{3}$ that may contribute to broken heart syndrome include:}
i. Epinephrine
ii. Duloxetine (Cymbalta)
iii. Venlafaxine (Effexor XR) and
iv. Levothyroxine.

Takotsubo cardiomyopathy is more commonly seen in postmenopausal women. ${ }^{12}$ Scientists believe one reason is that estrogen causes the release of catecholamine and glucocorticoid in response to mental stress.

The most common symptoms are chest pain, dyspnea and syncope.

Proposed Mayo Clinic criteria are required for the diagnosis

A. Echocardiographic transient left ventricular systolic dysfunction ( hypokinesis, akinesis or dyskinesis)

\section{B. Angiographic absence of obstructive coronary disease}

C. Electrocardiographic new ST-segment elevation and/or T wave inversion

\section{Absence of pheochromocytoma or myocarditis.}

Serum cardiac troponin levels are elevated in most patients (7.7 times the upper limit) while creatine kinase levels are generally normal or mildly elevated. The normal to mild elevation in creatine kinase contrasts with the risk of severe hemodynamic compromise. Levels of brain natriuretic peptide (BNP or pro-BNP) are elevated in more than $80 \%$ of patients. The treatment of Takotsubo cardiomyopathy is generally supportive in nature. Although patients with takotsubo heart disease may have low blood pressure, treatment with inotropes will usually exacerbate the disease. Since the disease is due to a high catecholamine state, patients should not be given inotropes. Treatment recommendations include intra-aortic balloon pump, fluids, and negative inotropes such as beta blockers or calcium channel blockers. Aspirin and other heart drugs also appear to help in the treatment of this disease, even in extreme cases.
Most of the patients survive the initial acute event, with a very low rate of in-hospital mortality or complications. Even when ventricular systolic function is heavily compromised at presentation, it typically improves within the first few days and normalises within the first two months. ${ }^{13,14}$ It is not likely for the same recovered patient to experience the syndrome twice. The average ages at on set are between 58 to 75 years affecting between $1.2 \%$ and $2.2 \%$ of people in Japan and $2 \%$ to $3 \%$ in western countries (Figures 1-4).

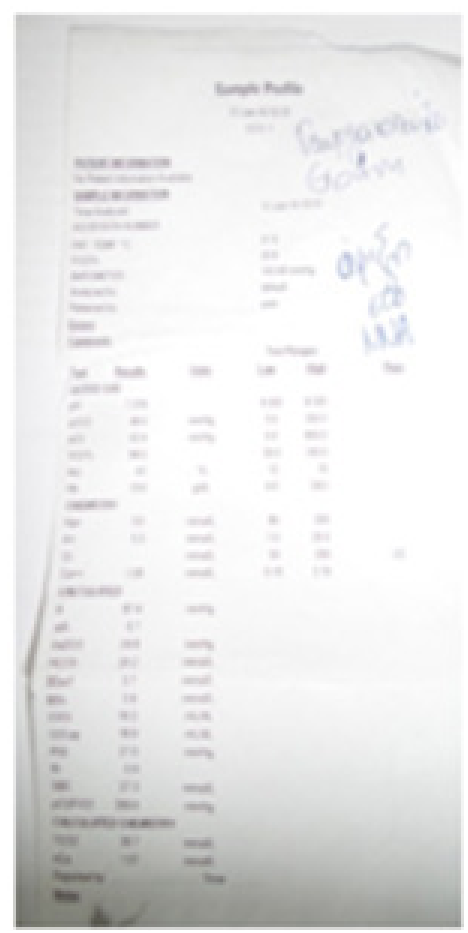

Figure I PI ABGs at arrival.

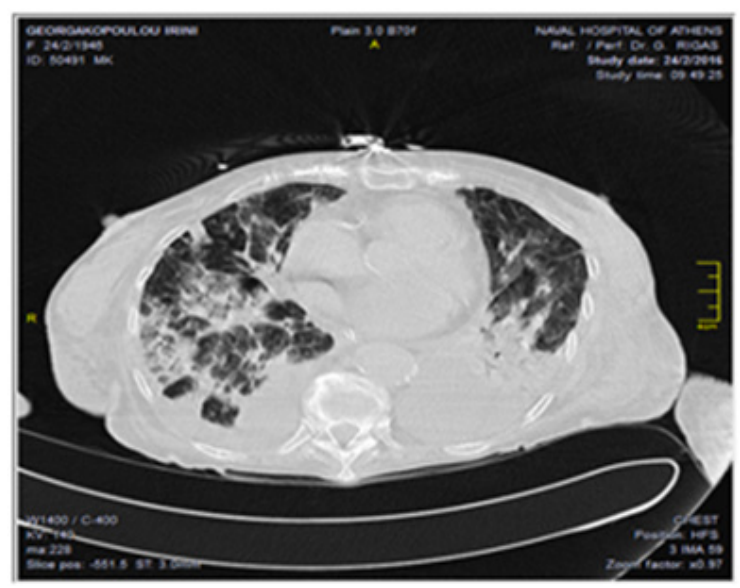

Figure 2 P2 CT scan.

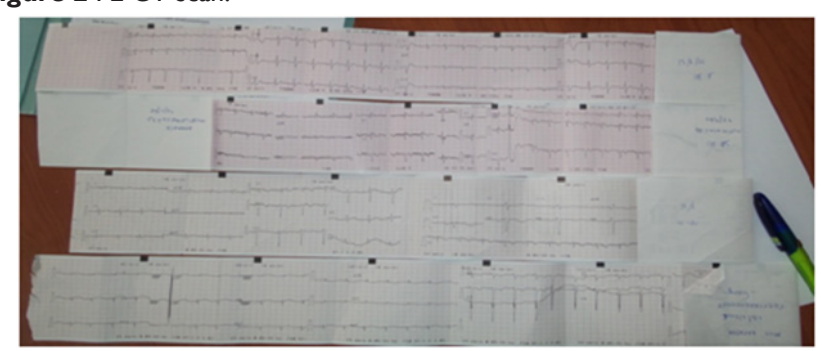

Figure 3 P3 ECG. 


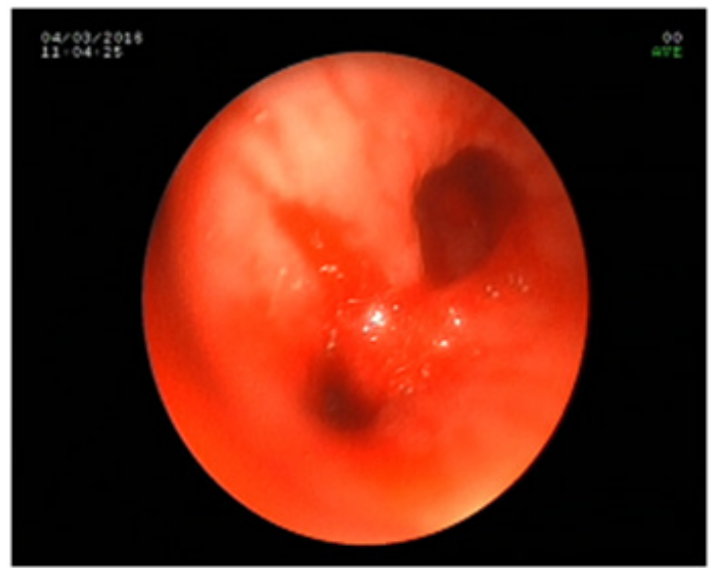

Figure 4 P4 Bronchoscopy.

\section{Acknowledgments}

None.

\section{Conflicts of interest}

The authors declare there is no conflict of interests.

\section{Funding}

None.

\section{References}

1. Mayo clinic research reveals 'broken heart syndrome' Recurs In 1 Of 10 Patients". Medical News Today, UK: MediLexicon International Ltd; 2006:737-742.

2. Sharkey S, Lesser J, Maron B. Takotsubo (stress) cardiomyopathy. American Heart Association. Circulation. 2011;124:e460-e462.

3. Wittstein IS, Thiemann DR, Lima JA, et al. Neurohumoral features of myocardial stunning due to sudden emotional stress. $N$ Engl $J$ Med. 2005;352(6):539-548.
4. Eshtehardi P, Koestner SC, Adorjan P, et al. Transient apical ballooning syndrome - clinical characteristics, ballooning pattern, and long-term follow-up in a Swiss population. Int J Cardiol. 2009;135(3):370-375.

5. Bergman BR, Reynolds HR, Skolnick AH, et al. A case of apical ballooning cardiomyopathy associated with duloxetine. Ann Intern Med. 2008;149(3):218-219.

6. Ibáñez B, Navarro F, Farré J, et al. Takotsubo syndrome associated with a long course of the left anterior descending coronary artery along the apical diaphragmatic surface of the left ventricle. Rev Esp Cardiol. 2004;57(3):209-216.

7. Inoue $\mathrm{M}$, Shimizu M, Ino $\mathrm{H}$, et al. Differentiation between patients with takotsubo cardiomyopathy and those with anterior acute myocardial infarction. Circ J. 2005;69(1):89-94.

8. Kurisu S, Sato H, Kawagoe T, et al. Tako-tsubolike left ventricular dysfunction with ST-segment elevation: a novel cardiac syndrome mimicking acute myocardial infarction. Am Heart J. 2002;143(3):448455 .

9. Tsuchihashi K, Ueshima K, Uchida T, et al. Transient left ventricular apical ballooning without coronary artery stenosis: a novel heart syndrome mimicking acute myocardial infarction. Angina PectorisMyocardial Infarction Investigations in Japan. $J$ Am Coll Cardiol. 2001;38(1):11-18

10. Desmet WJ, Adriaenssens BF, Dens JA. Apical ballooning of the left ventricle: first series in white patients. Heart. 2003;89(9):1027-1031.

11. Merli E, Sutcliffe S, Gori M, et al. Tako-Tsubo cardiomyopathy: new insights into the possible underlying pathophysiology. European Journal of Echocardiography. 2006;7(1):53-61.

12. Azzarelli S, Galassi AR, Amico F, et al. Clinical features of transient left ventricular apical ballooning. Am J Cardiol. 2006;98(9):1273-1276.

13. Akashi YJ, Nakazawa K, Sakakibara M, et al. The clinical features of takotsubo cardiomyopathy. QJM. 2003;96(8):563-573.

14. Nyui N, Yamanaka O, Nakayama R, et al. Tako-Tsubo' transient ventricular dysfunction:a case report. Jpn Circ J. 2000;64(9):715-719.

15. Branca P, Mc Gaw P, Light R. Department of medicine, vanderbilt university and saint thomas hospital. nashville, tennessee, USA Chest. 2001;119(2):537-546. 